\title{
Identification of Fake Video Using Smart Contracts and SHA Algorithm
}

\author{
SwapnaliTambe ${ }^{\mathrm{a}}$, Dr. Anil Pawar ${ }^{\mathrm{b}}$, Dr. S K Yadav ${ }^{\mathrm{a}}$ \\ ${ }^{a}$ Research Scholar, JJTU University, Rajasthan, India \\ ${ }^{b}$ Dean Academic Sanjivani COE, Kopargaon, India
}

\begin{abstract}
Deepfake is as a matter of fact a medium where one individual is supplanted by another who appears as though him. The profound bogus demonstration has been continuing for quite a long while. Profound phony uses incredible strategies, for example, AI and man-made consciousness to create and control visual and sound substance with high potential for the gadget. Profound misrepresentation relies upon the sort of impartial association called and the programmed encoder. These are essential for an encoder, which lessens a picture to a lower dimensional ideal and an ideal introduction picture. I examined various answers on various advances via web-based media stages like twitter and face book. From these examinations we are roused to extend this objective. In our proposed framework, we centre around identifying profound phony recordings utilizing blockchains, keen agreements, and secure hashing calculations. We utilize a few calculations to relieve the issue, for example, the SHA string
\end{abstract}

Keywords: Block Chain, Smart Contracts, SHA Algorithm

\section{Introduction}

Deepfakes are horrible for security, the confirmation of society and well-known government. At the point when this peril was introduced, methods for recognizing deepfake were proposed. Early undertakings relied upon made features got from abnormalities and sham video association relics. In relationship, continuous procedures have applied significant sorting out some way to thusly isolate critical and one-sided characteristics to recognize significant disfigurements [1]. A large portion of the present warmth exchangers are changing to a just progressed arrangement with no paper support being saved. This has ideas for dependability, check and provenance in various zones, like the arraignment, where the two players should be happy with the trustworthiness of the high level test, or occupied with security, where cases can be productive or come up short. In especially dependable terms and conditions; you may have to know precisely what the terms and conditions were at the hour of the arrangement. You in addition need to check the conditions of a game plan that were applied when the arrangement was concurred and set the essential creation dates when copyright issues emerge concerning mechanized substance. Moreover, there are ensured necessities for setting up earlier information prior to consenting to secret courses of action [09, 10]. An immense piece of this is that the crude information

\footnotetext{
${ }^{1}$ swapnali N Tambe, Research Scholar, JJTU University, Rajasthan, India Email: swapnali4231@gmail.com
} 
substance of a chronicle can be checked in any case, when the metadata is changed because of moving the record between working designs or contraptions. Summed up and feasibly open contraptions have gotten fundamental for video blend. Guaranteeing the authenticity of video occupations, for instance in court, acknowledgment offices, advancements, and the universe of diversion is basic. Thusly, there is a ton of investigation in the space of video approval and position adjustment systems. Moreover, research is in progress utilizing Darwin's information structure configuration to guarantee the unwavering quality of cutting-edge appropriation applications $[2,11]$. While there are various open apparatuses for gathering, encoding and isolating data, there are respectably not many open in the space of unwavering quality, check, and provenance confirmation. For example, you make and issue a verification that contains a SHA 256 hash assessment of the sent media close by customer nuances and a timestamp. This confirmation, hence, is affirmed by a high-level presentation gave by the essential Comodo authentication authority. Regardless, the issue here is that the principal report and the attestation are discrete components and could be easily detached while scattering or flowing the record [3, 4 and 18].

\section{Review of Literature}

In [01], display that a modernized underwriting relating to an image record can be set in that image archive close by the attached metadata containing references to the capable association. Despite the assortments among devices and between working structures and applications, a JPEG record holds its plan well in general. Right when changes occur, they by and large occur in the metadata locale and don't impact the data of the encoded picture, which is the center of the record and the part that ought to be sure. References to the capable association can be implanted in the archive's metadata. There is the advantage of having the high-level confirmation as a key piece of the archive it applies to and travel with the record suitably. Finally, we show that the metadata inside an archive offers the likelihood to join data that can be used to exhibit the uprightness, believability and provenance of the high-level substance inside the record.

The paper [02], clarify how deepfakes are a genuine danger to society, the type of government and organizations since they put focus on columnists who battle to channel the genuine news from the phony, they compromise security spreading promulgation that meddles with decisions, thwarting residents' trust in data given by specialists, and disposing of digital protection issues for people and associations. This examination territory expresses that there are numerous elements included that decide the idea of deepfakes. Contentions can be made, both for and against, however they may be basic if improvement and admittance to deepfake age apparatuses are appropriately administered. On the off chance that firmly checked, deepfakes can be utilized to help current mankind as opposed to making it fall.

FIPS [03], this article explains the Secure Hash Signature standard, four secure hash computations are shown in this standard: SHA-1, SHA-256, SHA-384 and SHA512 , to enlist a thick depiction of electronic data (message). Exactly when a message of any length $<264$ bits (for SHA-1 and SHA-256) or $<2128$ bits (for SHA-384 and SHA512 ) is gone into a computation, the result is a yield called the message digest. Message digests change long from 160 to 512 pieces, dependent upon the estimation. Secure hashing computations are conventionally used with other cryptographic estimations, as 
modernized mark computations and keyed hash message check codes, or in the time of sporadic numbers (bits). The four hashes showed in this standard are called protected considering the way that, for a given estimation, it is computationally hard to 1) find a message that facilitates with a given audit of the message, or 2) find two special messages that produce a comparative synopsis of the message. Any movements in a message will, with a particularly high probability, achieve another message digest. This will achieve an affirmation goof when the ensured hashing computation is used with a high-level imprint estimation or a keyed hash message approval computation.

The article [04, 08, 12], presented a blockchain-based response for modernized video validity testing in which secure and trustworthy perceptibility to the primary video. This print course of action can help fight deepfake video and sound by helping customers with choosing if video or progressed substance is unmistakable to a genuine and reliable source. If a video or mechanized substance can't be followed, the highlevel substance can't be trusted. Our sagacious agreement-based game plan gives discretionary skilled workers a strong strategy to request approval from the main specialist to copy and modify chronicles.

This article [05], focused in on the entertainment and replacement of human deepfakes. This article gives a start to finish assessment of how these headways work, the divergences between their designs, and how is being managed recognize them. We believe this information will be helpful to the neighborhood cognizance and thwarting malicious deepfakes.

The paper [07, 15], profound learning has been viably applied to deal with a couple of complex issues going from tremendous data examination to PC vision to human level control. Regardless, moves in significant learning have moreover been used to create programming that can make risks security, dominant part rule government and public wellbeing. One such significant learning application that has actually emerged is deepfake. Deepfake's computations can make fake pictures and accounts those individuals can't perceive from real ones. Thusly, proposing propels that can normally recognize and survey the uprightness of mechanized visual media is basic. This article presents an examination of the computations used to make deepfakes and, even more essentially, the techniques proposed for distinguishing deepfakes to date.

\section{Proposed System}

Our proposed framework appeared in Figure 1 fundamentally our framework completed in three distinct advances: administrator login, client login, programmer login. In this paper our emphasis is on the client login and programmer login, where the framework accepts the video as information and cycles that video for required yield. Through client login we are transferring credible video utilizing secure hash calculation. 'Secure Hash Algorithm' (SHA) is a gathering of cryptographic hash limits made by the US 'Public safety Agency' (NSA) and disseminated as a standard by the US 'Public Institute of Science and Technology' (NIST). It is the crucial estimation for secure applications used by US government associations. A critical component of SHA estimations is that they do an intensifying wonder, which infers that a little change in the data achieves a tremendous change in the yield regard [16]. 
No

Yes

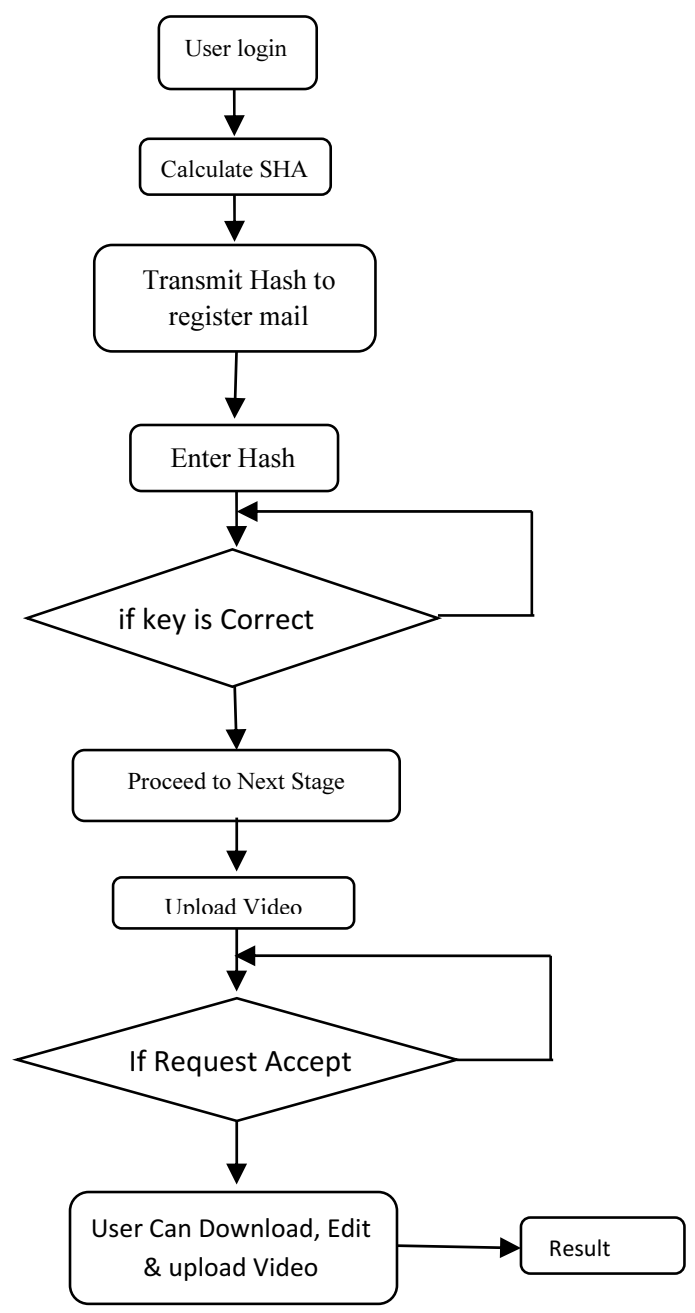

Figure 1. Proposed system for the smart contract

The main variation, SHA-1, makes a 160-piece regard and was conveyed in 1993; regardless, it was taken out not long after appropriation due to an undisclosed shortcoming and a changed structure was conveyed two years afterward. In 2002, NIST conveyed the SHA-2 gathering of limits. Not in any way like SHA-1 with a fixed 160piece hash size, SHA-2 is offered in six structures that produce an extent of yield sizes from 224 to 512 pieces, the most ordinarily used being SHA-256 and SHA-512. Like MD5 and SHA-1, SHA-2 limits rely upon the Merkle-Damgård advancement. The computation used for SHA-256 is:

1. A 256-bit data support is made, included 8 to 32-digit words which are taken care of with the underlying 32 bits of the incomplete bits of the square hidden establishments of the underlying 8 primes [01, 03 and 17]. 
2. A 64 segment table of constants is prepared using the first 32 bits of the fractional bits of the 3D shape establishments of the underlying 64 Primes [01, 03 and 17].

The data is padded with a primary piece "1" and the length of the main data is conveyed as a 64-cycle number, segregated by the number of zeros expected to make the message length, including padding, an alternate of 512 - little [01,03 and 17].

3. Each 512-cycle block is taken care of through 64 rounds where each round incorporates a movement of exercises included bitwise undertakings and estimated extension [01, 03 and 08].

4. The assessment of the pad on fulfilment of each square is the fundamental impetus for the going with square; around the completion of the last square, the support contains the hash regard [01,03 and 17].

While transferring a credible video as demonstrated in the flowchart, we should initially utilize the client login through which a SHA is determined and a hash key is created which is shipped off a valid email ID which is a brilliant agreement. The primary expert sharp arrangement is made using attributes, for instance, the owner having the Ethereum address of the main specialist and mappings containing video detail records reliant on the circumstance with assents permitted or denied. Moreover, regardless of sales are put something for reference and history following. In like manner, a critical once-over that guides in conspicuousness is the overview of designated authentication accounts which are seen as assistant chronicles of the principal understanding. Savvy contracts are robotized programs that encode conditional auto-execution rationale and are implemented utilizing decentralized encryption strategies. Notwithstanding their name or legitimate status, brilliant agreements produce extraordinary premium and venture since they can fundamentally change how the gatherings cooperate. Savy Contract makes the first craftsman video. In this arrangement, an understanding is made for the other individual who wishes to acquire consent to change, adjust and even circle these recordings identifying with the $\mathrm{T}$ and $\mathrm{Cs}$ gave in the arrangement. This solicitation for understanding is put away on the interplanetary record framework worker. Hash planning is accessible in the savvy contract. Someone else demands a solicitation to alter, share or alter this video content. At the point when an auxiliary craftsman demands the proprietor, it implies that they affirm the terms and states of the arrangement $[13,14]$. The solicitation is gotten by the proprietor and afterward the yield is shown. This arrangement not exclusively can take various consent at time yet in addition can taking different solicitation sent from same proprietor. At the time proprietor affirms the application, they made the youngster contract thought about at the underlying arrangement and they update parent's information. Now, the another proprietor, request a consideration of their as of late maded arrangement through beginning proprietor by contact of the main video. At same time, beginning proprietor point supports and grants a validation at that point noticing an as of late made SC. An adequately affirmed shrewd agreement would by then be incorporated the kid inside a remarkable SC. They address the Ethereum of the other segment of their qualities appeared in Figure 2 stream graph. 
Yes

No

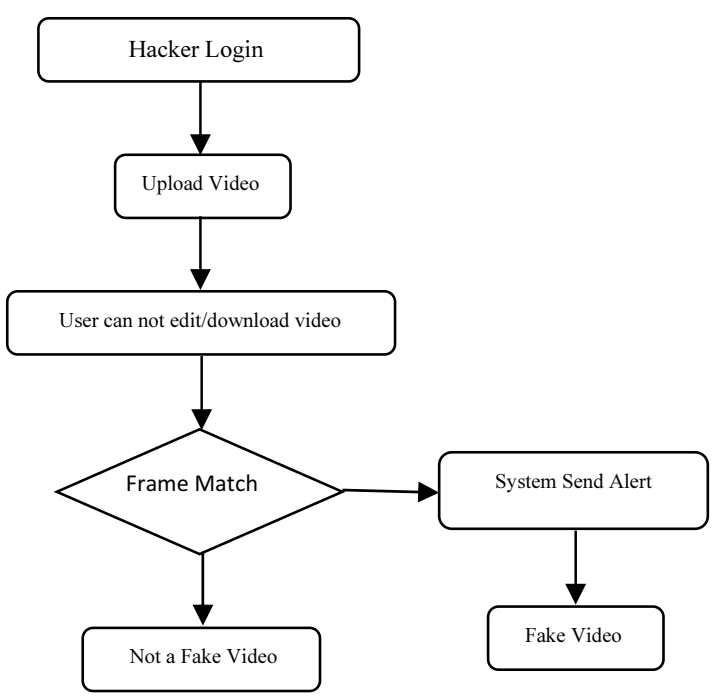

Figure 2. System for the identification of video

\section{Testing}

Shrewd Contract makes the first craftsman video. In this understanding, the arrangement is made for the other craftsman for whom you wish to get consent to adjust, alter and even course these $\mathrm{T} \& \mathrm{C}$ related recordings gave in the arrangement. This solicitation for understanding is put away on the interplanetary record framework worker. Hash planning is accessible in the keen agreement. Another proprietor demands a solicitation to alter, share or alter this video content. At the point when an optional craftsman demands the ownerit suggests that they confirm the terms and conditions of the plan. The solicitation is gotten by the proprietor and afterward the yield is shown. Not exclusively would this be able to contract demand various consents simultaneously, it can likewise acknowledge different solicitations sent by a similar proprietor. Right now the proprietor affirms the solicitation, has gone into the agreement for the youngster as for the underlying agreement and updates the information of the guardians. Now, the other proprietor demands a consideration of their recently closed agreement through the underlying proprietor by means of the contact of the main video. Simultaneously, the underlying proprietor point backs up and ensures validation, at that point takes a gander at a recently made SC. A keen agreement really affirmed around then would incorporate the youngster inside a solitary SC. They go to Ethereum on the opposite side of your traits.Smart contracts make a connection between unmistakable substances, the SC is the main proprietor is a proprietor who has that Ethereum address in the underlying proprietor and tallies until he keeps the video records. Additionally, all solicitations are put something aside for history following and following. In the extension, a fundamental rundown has an effect in the discoverability rundown of permitted affirmation enlistments that are viewed as optional enrollments of the underlying agreement. Each agreement comprises of a video. In this manner, 1: 1 is the connection between the arrangement and the substance of the video. Besides, every video is connected as though it were an 
Ethereum address with a proprietor. Moreover, a tricky arrangement can have a few kid contracts dependent on effective verification. Thusly, a 1: $\mathrm{N}$ association between the mischievous proprietor of the underlying understanding. At last, the Interplanetary File System is likewise a substance with a 1: 1 association with any SC, every video is moved to the Interplanetary File System workers, and its Interplanetary File System hash is a property inside the SC. Moreover, the T\&C understanding structure of each agreement is passed notwithstanding the interplanetary document framework worker and its hash is a quality inside the keen agreements made for enlistments. Moreover, the complete code available to all inconspicuous components was additionally made. Following pictures are some Hash kay produced test appeared in Figure 3,

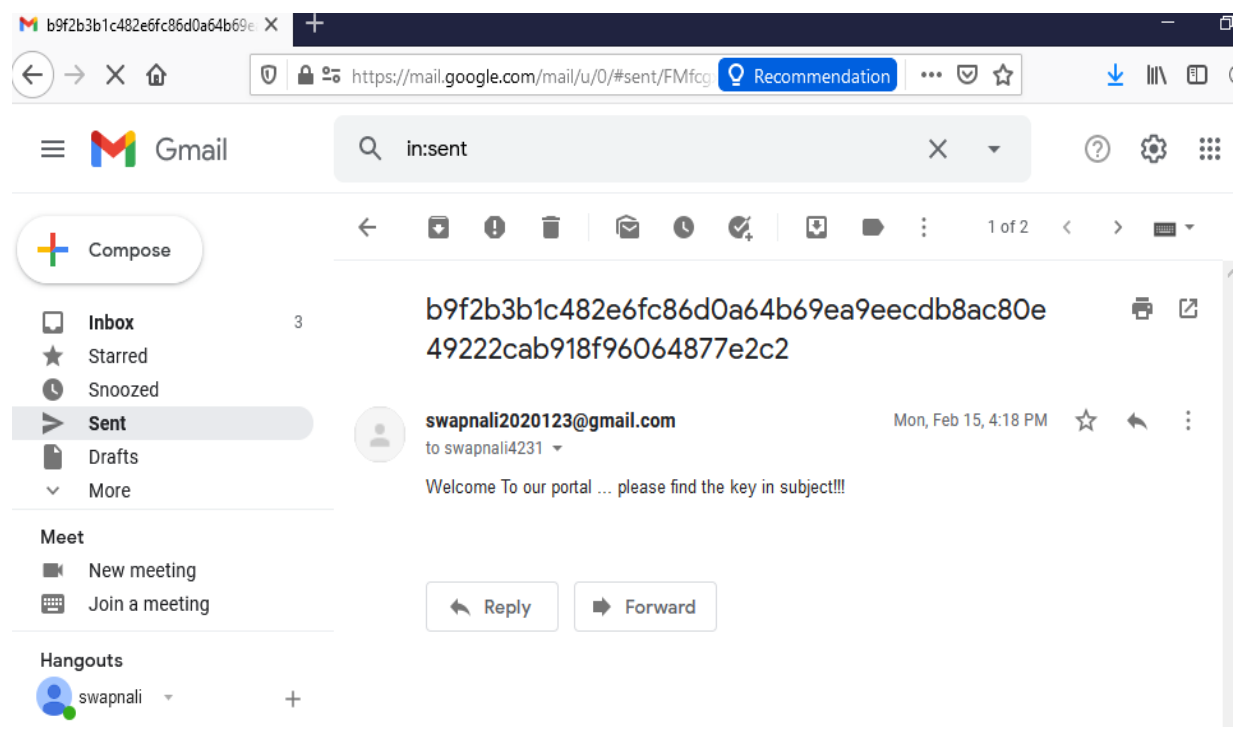

Figure 3. Hash Kay send to email

\section{Conclusion}

Free admittance to make and share data that has no realities behind it via online media stages like WhatsApp and other advanced stages has uncovered another issue of bogus data, which has produced bits of hearsay all throughout the planet. In this paper, we have introduced a climate arrangement of an Ethereum blockchain-based solution for confirmation of verify of advanced recordings where secure and dependable discernibility can be set up to the maker or wellspring of the first video, in a totally decentralized. Our answer permits web-based media clients to approach believed information from computerized content so they can follow the information and have certainty that the information is genuine. The arrangement utilizes Ethereum shrewd agreements and IPFS decentralized capacity framework. The Ethereum wallet executes keen agreements for recordings and IPFS is utilized to store the metadata of the recordings and furthermore creates an extraordinary hash of the recordings to find the documents in IPFS. The development of our proposed plan, system arrangement, progression diagrams and execution nuances can be applied to any automated substance like video and pictures. This Smart Contract-based game plan gives 
discretionary experts a strong strategy to request assent from the primary specialist to copy, change, share and adjust chronicles. Work is in progress to plan and do a totally valuable and operational decentralized standing system. At this moment, the arrangement for the Ethereum framework, savvy arrangements and private chain has been successfully made.

\section{References}

[1] Martin Harran, William Farrelly, Kevin Curran, "A method for verifying integrity \&authenticating digital media", Applied Computing and Informatics, 2017

[2] Hrisha Yagnik1, Akshit Kurani2, Prakruti Joshi3, "A Brief Study on Deepfakes", IRJET, Volume-7 Issue-12, DEC 2020.

[3] FIPS, "Secure Hash Standard",Federal Information Processing Standards Publication 180-2, 01 Aug 2002

[4] Haya R. Hasan And Khaled Salah, "Combating DeepfakeVideos Using Blockchainand Smart Contracts"IEEE Access April 12, 2019, Digital Object Identifier 10.1109/ACCESS.2019.2905689.

[5] YisroelMirsky,Wenke Lee, "The Creation and Detection Of Deepfakes: A Survey", ACMComputing Surveys, Vol. 1, No. 1, January 2020

[6] Alok Chauhan, Amit Kumar,"Establishing Environment Setup for Preventing DeepfakesusingBlockchain Technology",MuktShabd Journal, Volume IX Issue V, $\mathrm{MAY} / 2020$.

[7] Thanh Thi Nguyen, Cuong M. Nguyen, Dung Tien Nguyen, Duc Thanh Nguyen, SaeidNahavandi, "Deep Learning for Deepfakes Creation andDetection: A Survey", aXiv: $2019.11573 v 2$ [cs.CV].Jul 2020

[8] Paula Fraga-Lamas, Tiago M. Fern'andez-Caram'es. "A Review on the Use of Blockchain for the Internet of Things",IEEE Access, Electronic ISSN: 2169-3536, 31 May 2018.

[9] H. Allcott, "Social Media and Fake News in the 2016 Election",Journal of Economic Perspectives, Vol. 31, No.2 Spring 2017.

[10] R. Chesney and D. K. Citron., "Deep Fakes: A Looming Challenge for Prives: A Looming Challenge for Privacy, Democr, Democracy, and National Security", California Law Review, Vol. 107:1753 2018

[11] Potharaju, S. P. (2018). An Unsupervised Approach For Selection of Candidate Feature Set Using Filter Based Techniques. Gazi University Journal of Science, 31(3), 789799.

[12] P. Treleaven, R. G. Brown, and D. Yang, "Blockchain technology in finance," Computer, no. 9, pp. 14-17, 2017.

[13] F. Tian, "A supply chain traceability system for food safety based on haccp, blockchain\& internet of things," in Service SystemsandServiceManagement(ICSSSM), 2017InternationalConferenceon. IEEE,2017, pp. 1-6.

[14] Potharaju, S. P., \& Sreedevi, M. (2017). A Novel Clustering Based Candidate Feature Selection Framework Using Correlation Coefficient for Improving Classification Performance. Journal of Engineering Science \& Technology Review, 10(6).

[15] M. A. Khan and K. Salah, "IoT security: Review, block chain solutions, and open challenges," Future Generation Computer Systems, vol. 82, pp. 395-411, 2018.

[16] K. Biswas and V. Muthukkumarasamy, "Securing smart cities using block chain technology," in 2016 IEEE 18th International Conference on High Performance Computing and Communications; IEEE 14th International Conference on Smart City; IEEE 2nd International Conference on Data Science and Systems (HPCC/SmartCity/DSS), Dec 2016, pp. 1392-1393. 
[17] K.Christidis and M. Devetsikiotis, "Block chains and smart contracts for the internet of things," IEEE Access, vol. 4, pp. 2292-2303, 2016.

[18] Swapnali N. Tambe, A.B.Pawar, "Detecting fake Videos Using Block Chain and Smart Contracts”, IJRTE, ISSN: 2277-3878, Volume-8 Issue-4S5, December 2019. 